\title{
Ein neues Handbuch über die ungarische Sprachgeschichte
}

<https://doi.org/| 0.33339/fuf.79537>

Kiss Jenő \& Pusztai Ferenc: $A$ magyar nyelvtörténet kézikönyve [Handbuch der ungarischen Sprachgeschichte]. Budapest: Tinta könyvkiadó 2018. $548 \mathrm{~S}$.

Das von Jenö Kiss und Ferenc Pusztai herausgegebene Handbuch der ungarischen Sprachgeschichte bietet den Lesern neue Perspektiven auf die Geschichte der Sprache. Obwohl das Werk von der Seitenzahl her nicht besonders umfangreich ist - einschließlich der Anhänge zwar über 500 Seiten, doch es hat schon umfangreichere gegeben - bietet es eine ganzheitlichere Annäherungsweise an die Geschichte der Sprache als seine Vorgänger, indem es auch die auBersprachliche Geschichte und die soziolinguistische Entwicklung der letzten Jahrhunderte berücksichtigt. Im Kern des Sprachwandels stehen der Sprachbenutzer und die Sprachgemeinschaft.

Das Ungarische ist infolge seiner langen schriftlichen Geschichte ein dankbares Objekt für die Untersuchung des Sprachwandels. Schon auf den ersten Seiten werden als Beispiele Fragmente aus neun Bibelübersetzungen aus 600 Jahren angeführt. Dies ist ein hervorragender Schnellkurs über die konkrete Veränderung der geschriebenen Sprache. Selbst ein flüchtiger Vergleich macht vieles sichtbar.

In der zweiten Hälfte der Einleitung werden Fragen zur Veränderung der Sprache angeführt und beantwortet. Man wüsste gern, woher die Fragen stammen, denn in vielen geht es allgemein um den Sprachwandel, doch es sind auch einige überraschende Fragen darunter. Dieser Teil bietet eine vergnügliche Lektüre, weckt aber die Frage, für wen das Buch eigentlich gedacht ist. Für ein Lehrbuch sind die Fragen angemessen, aber an welches $\mathrm{Pu}$ blikum will sich das „Handbuch“ richten? Unter den überraschenden Fragen sei diejenige erwähnt, in der es darum geht, ob die Beschleunigung des Sprechtempos mit dem Sprachwandel verknüpft ist. Die Verfasser verweisen tatsächlich auf eine Untersuchung, der zufolge das Sprechtempo des Ungarischen in den letzten Jahrzehnten gestiegen sei. Konkrete Beispiele werden nicht gegeben, obwohl der Einfluss des Tempos auf die Veränderung zugestanden wird. Auf die Frage ,ist das Ungarische eine europäische oder 
eine europäisierte Sprache" wird am Ende des Buches und auch dieser Rezension eingegangen.

Das zweite Kapitel über die ungarische Sprachgeschichte als Gegenstand der Forschung (S. 43-98) enthält viele bekannte grundlegende Fakten. In diesem Kapitel werden die sprachgeschichtlichen Stufen des Ungarischen vorgestellt und gleich zu Beginn für die Länge der vorungarischen Stufe zwei Alternativen genannt, von denen sich die eine auf die traditionelle Auffassung und die andere auf die neuere Lehnwortforschung stützt. Die Variationsweite beträgt ca. 500 Jahre. Als Instrumente der sprachgeschichtlichen Forschung werden schriftliche Dokumente, das Paradigma der scheinbaren Zeit sowie die Erforschung der verwandten Sprachen und der Kontaktsprachen angeführt. Über die ungarischen Sprachdenkmäler informiert eine separate Darstellung von Erika Terbe, der Überblick über die Entwicklung der Orthografie stammt von Klára Korompay.

Das dritte Hauptkapitel bietet eine Darstellung der ungarischen Sprachgeschichte aus traditioneller Sicht. Die von Károly Gerstner verfasste kompakte Beschreibung der Konsonant- und Vokalgeschichte (S. 103-128) folgt den Stufen der Sprachgeschichte und stellt die bekannten lautgeschichtlichen Veränderungen und Tendenzen dar.
Zsófia Sárosi hat die Darstellung der Morphemgeschichte verfasst (S. 129-153), in der auf allgemeiner Ebene die morphologische Entwicklung der Wortstämme sowie der Ableitungssuffixe und der Flexionszeichen und -endungen beschrieben wird. Auch dies ist aus vielen früheren Quellen bekannt. Unter anderem wird der Ursprung sowohl der Personalendungen der Verben als auch der Possessivsuffixe der Nomina mit der Agglutination der Pronomina und die Arbeitsteilung der Endungen der Konjugation mit der Vermeidung von Homonymie erklärt. Dieser Abschnitt bietet wenig Neues, da ein großer Teil der Elemente der Flexionsmorphologie nicht im Einzelnen erklärt wird. Insofern unterscheidet er sich deutlich z.B. von der vorangehenden Darstellung der Lautgeschichte. Der Geschichte der Wortklassen ist ebenfalls ein eigener Abschnitt gewidmet (verfasst von Attila Hegedűs und Mária D. Mátai), in dem die frühesten und späteren Stadien der verschiedenen Wortklassen dargestellt werden.

Anschließend wird ausführlich die Geschichte der Syntax behandelt (S. 168-225, verfasst von Magdolna Gallasy, Lea Haader und László Horváth), was durch die Betrachtung von Textdenkmälern aus verschiedenen Zeiten fruchtbar ist und detailliert geschieht, 
sowie die Geschichte der Texte (S. 226-246, verfasst von Magdolna Gallasy), wobei die Genres sowohl vorliterarischer als auch literarischer Texte betrachtet werden. Die Darstellung der Geschichte der Lexik ist traditionell, und es hat den Anschein, dass die Lehnwortforschung im Bereich des Ungarischen in den letzten Jahrzehnten keine solchen grundlegenden Veränderungen ausgelöst hat wie z.B. im Fall des Ostseefinnischen. Zur Darstellung der Geschichte der Lexik gehören auch Abschnitte über die Bedeutungsveränderungen ( $\mathrm{S}$. 271-283, Ferenc Pusztai) und über die Geschichte der Eigennamen (S. 283-307, Mariann Slíz).

Die beiden letzten Hauptkapitel des Buches befassen sich mit Themen, die in den Standardwerken und Lehrbüchern zur Sprachgeschichte bisher weniger behandelt wurden, nämlich mit der Geschichte der Sprachvariation und der ungarischen Sprachgemeinschaft. Die Sprachvariation wurde bisher kaum aus sprachgeschichtlicher Perspektive behandelt.

Das erste Unterkapitel über die Geschichte der Sprachvariation befasst sich mit der Geschichte der Dialektvariation (Dezső Juhász, S. 314-349). Obwohl Dialekte gesprochene Sprachformen sind, werden auch sie vornehmlich anhand von Sprachdenkmälern behandelt.
Die Anzahl der Beispiele für die vorungarische Dialektvariation ist gering. In diesem Unterkapitel werden überraschend genau einige Besonderheiten der Morphologie behandelt, die ich im früheren Teil über die Geschichte der Morphologie erwartet hatte: nämlich die Entwicklung der vieldiskutierten Formen der 1. Person Plural des Präsens und der 3. Person Singular des Imperfekts der bestimmten Konjugation. In diesem Zusammenhang dienen sie als Beispiele für die Dialektvariation, und die heutige Vertretung wird unter den Ziffern 12 und 13 der Kartenbeilage dargestellt. Im Bereich der Morphologie wird auch angemerkt, dass die sog. Familien-Lokalkasus (-nott, -nól, -nyi) zwar erst in der Zeit des Mittelungarischen in schriftlichen Dokumenten begegnen, wahrscheinlich aber auf das Vorungarische zurückgehen. Die Begründung für diese Datierung wird freilich nicht genannt; der Grund ist ja, dass diese Lokalkasusendungen Entsprechungen in den obugrischen Sprachen haben. Die Fragen der Dialektvariation in den anderen sprachgeschichtlichen Stadien des Ungarischen, insbesondere die Geschichte und Variation der Vokale, werden anhand der Sprachdenkmäler detailliert dargestellt.

Den Überblick über die Entwicklung der Standardsprache hat 
Jenő Kiss verfasst (S. 350-377). Zu Beginn werden vielleicht etwas überraschend die Berufssprachen behandelt. Der Anlass dafür scheinen die in historischen Quellen und Sprachdenkmälern begegnenden Berufsbezeichnungen zu sein, die zu einem großen Teil in Familiennamen auftreten. Über die Entwicklung der Sprache sagt diese Terminologie ihrer eigenen Zeit jedoch nicht allzu viel aus. Als Teil der Berufssprachen wird kurz auch auf die Entwicklung der Wissenschaftssprache eingegangen. Dabei werden die seit Jahrhunderten anhaltende bewusste Entwicklung der Sprache und die Wichtigkeit der ungarischsprachigen Terminologie und des wissenschaftlichen Begriffsapparats betont. Die eigentliche Entwicklung der Standardsprache wird wieder in der Reihenfolge der Epochen der Sprachgeschichte behandelt, in denen jeweils eigene außersprachliche Faktoren die Entwicklung der Sprache beeinflussten. Die bedeutendste Entwicklungsphase in der Zeit des Neuungarischen ist natürlich die sog. Sprachreform um die Wende vom 18. zum 19. Jahrhundert, eine Epoche der eifrigen bewussten Weiterentwicklung der Sprache. Die Zeit der Sprachreform wird zwar auf zwei bis drei Seiten behandelt, doch aus irgendeinem Grund bleibt die Behandlung oberflächlich und wenig gehaltvoll. Oder verhält es sich so, dass die Reform letztlich doch nicht so bedeutsam war, wie generell zu verstehen gegeben wurde? Insbesondere im Hinblick auf die Entwicklung der Standardsprache ist interessant, dass die Darstellung bis zum Zeitpunkt der Niederschrift, in das Jahr 2016 reicht. Bei der Entwicklung der jüngsten Zeit werden die Urbanisierung, die Binnenwanderung und der Slang hervorgehoben, während der Einfluss der Veränderung der Medien auf die Entwicklung der Standardsprache äußerst wenig Beachtung findet. Man würde annehmen, dass sie neben der Globalisierung ein mindestens ebenso wichtiger Faktor ist.

Das fünfte und letzte Kapitel des Buches befasst sich mit der Geschichte der Sprechergemeinschaft des Ungarischen (S. 382-415). Es beginnt mit einem allgemein gehaltenen Unterkapitel über Demografie, in dem die Geschichte der ungarischsprachigen Bevölkerung von der Einwanderung bis ins 21. Jahrhundert umrissen wird. Die demografische Entwicklung ist natürlich eng mit der staatlichen Geschichte Ungarns verknüpft, und die Gebietsverluste im Gefolge des Friedens von Trianon werden mehrfach erwähnt. Dieser Teil ist stark gesellschaftspolitisch gefärbt. Für die anderen Kapitel oder Unterkapitel wurden keine Mottos gewählt, 
doch diesem Abschnitt wurde ein Zitat aus einem Interview mit György Györffy (1989) beigefügt: „kein europäisches Volk ist in seinem Gebiet Ureinwohner". Dies führt den Leser unnötigerweise in nationalistische Gedankengänge. Das Unterkapitel enthält auch einen $\mathrm{Ab}$ schnitt, in dem der Begriff Ethnie erörtert wird. Die historische und kulturhistorische Entwicklung der ungarischen Sprachgemeinschaft wird im letzten Teil dargestellt. Bei der Beschreibung der Epoche des Vorungarischen werden zwei Punkte stark hervorgehoben: erstens, dass das Ungarische, weil es nach der Lösung aus der Verbindung mit den obugrischen Sprachen eine lange Phase der Differenzierung erlebt hat (dies wird in keiner Weise problematisiert; in Finnland vertreten ja viele Forscher eine andere Auffassung), zu den „älteren“ Sprachen des Gebiets gehört, verglichen z.B. mit den slavischen oder romanischen Sprachen. Zwar hat das Ungarische vor allem im Vergleich zu den anderen finnisch-ugrischen Sprachen eine ungewöhnlich lange Sonderentwicklung durchlaufen, doch die Bewertung von Sprachen aufgrund ihres "Alters" ist fragwürdig. Früher wurden gelegentlich Sprachen als alt bezeichnet, in denen viele archaische Züge erhalten geblieben sind. In gewisser Weise sind natürlich alle Sprachen gleich alt. Ein zweiter Umstand, der in diesem Kapitel erneut betont wird, ist, dass das Ungarische in Europa entstanden ist und nicht in Asien wie seine obugrischen Verwandten. Ein überraschend großer Teil der Betrachtung der Epoche des Altungarischen ist der Beziehung zwischen dem Ungarischen und dem Slowakischen gewidmet; mir ist nicht bekannt, an welche Diskussion dieser Abschnitt anknüpft. Man hat den Eindruck, dass es dafür einen Impuls außerhalb des hier $\mathrm{zu}$ besprechenden Werks gibt. Aus dem abschließenden Kapitel geht deutlich hervor, dass die ungarische Sprache seit der Einwanderung in den Sog vielerlei sprachlicher Kontakte geriet. Diese Information ist nicht neu, aber im Hinblick auf die Entwicklung der Sprache natürlich wichtig.

Das Buch enthält zahlreiche nützliche Anhänge: Das enzyklopädische Lexikon der Sprachgeschichte ist das erste seiner Art, das mir zu Gesicht gekommen ist. Dass es zusammengestellt wurde, dürfte darauf zurückzuführen sein, dass das Werk nicht nur als Hand-, sondern auch als Lehrbuch dienen soll. Die zahlreichen Karten sind übersichtlich. Auch ein Sachregister findet sich.

Wie gut erfüllt das Buch seinen Zweck? Das Inhaltsverzeichnis weckt mehr Erwartungen als der 
tatsächliche Inhalt erfüllt. Dies gilt besonders für die letzten Kapitel, die Informationen über bekannte außersprachliche Umstände wiederholen, ohne neue Überlegungen zu der Beziehung zwischen der Sprache und der außersprachlichen Geschichte zu bieten. Die lange schriftliche Geschichte des Ungarischen hätte mit ihren Dokumenten die Voraussetzungen dafür liefern können. Vielleicht will man den Leser selbst zu diesen Überlegungen anregen, da die entsprechenden
Themen einem Handbuch der Sprachgeschichte beigefügt wurden. Als Lehrbuch ist das Werk eine hervorragende Einführung in das Thema, auch wenn leider viele Grundlagen vor allem aus der Geschichte der Morphologie fehlen. Das gleiche gilt natürlich auch für das Werk als „Handbuch”: Nicht alles ist darin zu finden, und viele Dinge werden in den verschiedenen Teilen des Buchs aus unterschiedlichen Perspektiven behandelt.

Ulla-Maija Forsberg

\section{Von Sprachkontakten zum Sprachtod oder zur Superdiversität?}

Linguistic Genocide or Superdiversity? New and Old Language Diversities. Ed. by Reetta Toivanen and Janne Saarikivi. Linguistic Diversity and Language Rights: 14. Multilingual Matters. Bristol - Buffalo • Toronto 2016.362 S.

In der Uralistik ist man ständig damit konfrontiert, dass Sprachen, die wir untersuchen, bedroht sind und aussterben. Das Problem beschränkt sich nicht allein auf die uralischen Sprachen, denn unterschiedlichen Schätzungen zufolge sind 50-90 \% der Sprachen der
Welt vom Aussterben bedroht. Diese Entwicklung wurde vor allem seit dem 20. Jahrhundert durch die Kolonisation, die Modernisierung und die Globalisierung beschleunigt. Als Folge dieser Faktoren sind auch neue Kreolsprachen, Mischsprachen sowie neue ethnische Identitäten, Städter- und Einwanderergruppen entstanden. Die Welt hat sich verändert, und die Veränderung wird immer schneller. Dieser Prozess bringt nicht nur den Tod von Sprachen mit sich, sondern auch eine neuartige - und weitgehend unerforschte - ethni- 